NASA TECHNICAL NOTE

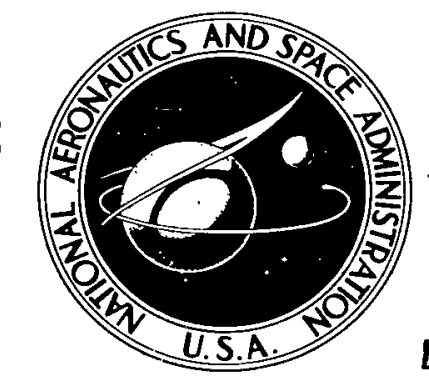

NASA TN D-4309

C. 1

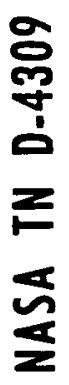

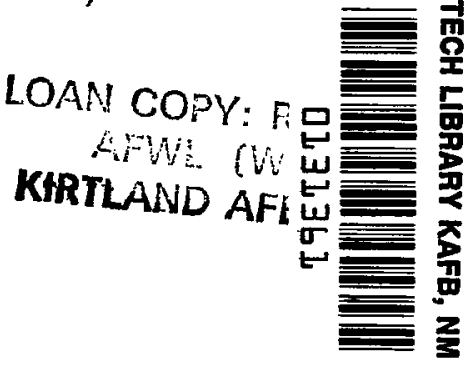

\title{
A STUDY OF THE POSSIBLE \\ USES OF PLASMA FOR \\ MILLIMETER WAVE GENERATION
}

by Robert E. McIntosh

Electronics Research Center

Cambridge, Mass.

NATIONAL AERONAUTICS AND SPACE ADMINISTRATION - WASHINGTON, D. C. - MARCH 1968 


\section{A STUDY OF THE POSSIBLE USES OF PLASMA}

FOR MILLIMETER WAVE GENERATION

By Robert E. McIntosh

Electronic Research Center

Cambridge, Mass.

\section{NATIONAL AERONAUTICS AND SPACE ADMINISTRATION}




\title{
A STUDY OF THE POSSIBLE USES OF PLASMA FOR MILLIMETER WAVE GENERATION
}

\author{
by Robert E. McIntosh
}

Electronics Research Center

\section{SUMMARY}

The millimeter wave portion of the frequency spectrum has not been utilized in communication systems because of a lack of practical, high-power $\mathrm{mm}$ wave generators. As conventional tube design techniques have been only moderately successful in extending the microwave power frequency frontier, a number of novel approaches for obtaining $\mathrm{mm}$ wave sources have been suggested. Two of these methods rely on the use of a gaseous plasma and are described in this technical note.

The electron beam-plasma interaction and the plasma multiplier have been investigated during the last decade in the hope that one or the other might have some application at $\mathrm{mm}$ wavelengths. So far the expectations for these devices have not been realized because of many unresolved engineering problems. This report describes the major difficulties that must be overcome before further development in this area can proceed, and it attempts to evaluate the potential of both devices. 


\section{INTRODUCTION}

High-power generation and amplification in the microwave frequency range (100 MHz to $100 \mathrm{GHz}$ ) has been achieved for some time. Well-known devices -- such as the traveling-wave tube, backward-wave oscillator, and magnetron -- have been useful in exploiting this frequency range for communications. Furthermore, recent advances in laser technology have led to the development of coherent sources at optical frequencies. However, no high-power source has yet been developed for the millimeter wave range between microwave and optical frequencies.

Attempts at extending microwave techniques to obtain millimeter wave sources have been successful to a point. At present, conventional devices that are of the periodic-circuit type dominate the microwave power/frequency frontier (ref. 1). In addition, microwave tubes of a more specialized nature have been investigated and promise to extend the upper frequency limit in the future (ref. 2). However, most of these tubes have disadvantages in that the fabrication and beam-formation techniques are impractical at millimeter wave frequencies, owing to the small sizes required. The heat transfer problems associated with the delicate circuitry also limit the output power of these devices.

Frequency multipliers that transfer energy from microwave to millimeter wave frequencies are being scrutinized by a large number of people at the present time. So far, the power handling capability of these devices is low. Although the conversion efficiency is potentially high for certain types of solid-state varactors, their output power has been unimpressive to date. As the state of the art advances in this area, more acceptable power levels may well be achieved. Until the inherent problems in tube and varactor design can be solved, an impasse therefore exists in the development of high-power millimeter wave sources.

A number of novel schemes have been proposed during recent years to circumvent the problems mentioned above. Some of these designs rely on the use of a gaseous plasma to replace the metallic, slow-wave structure of the conventional electron beam tube, or to act as a non-linear medium in a frequency multiplier. It is the purpose of this technical note to evaluate the potential of these devices for the generation of millimeter waves.

The devices that utilize a plasma to generate microwave or millimeter wave signals fall into two major categories:

1. Non-linear interaction devices (e.g., harmonic multipliers)

2. Beam-plasma interaction devices (e.g. , beam plasma amplifiers). 
Research is currently being carried on in each of these areas. This document describes the progress that has so far resulted from these investigations. The obstacles that have to be surmounted before these devices will find practical application as millimeter wave sources are also emphasized. Finally, the most promising approaches to the development of useful generators are evaluated.

Harmonic multipliers that use the non-linear properties of a plasma to obtain frequency multiplication are discussed in section II. In section III, a summay of recent developments in the area of beam plasma devices is presented. In each case an attempt has been made to describe the physical phenomena involved. In section IV comments on some of the limitations that plasma multipliers and beam-plasma devices have at millimeter wave frequencies are discussed. In that section, the engineering problems that have to be solved are summarized. The references present a fairly complete list of work done in these areas.

The guidance given the author in this investigation by Messrs. C. M. Veronda, B. Kulke, and W. H. Kohl, and the technical discussions with Messrs. P. Chorney and C. B. Swan are here acknowledged. 


\section{PLASMA HARMONIC GENERATORS}

It has been proposed that one non-linear medium which can dissipate the required power for large-signal millimeter wave multipliers is the gas discharge (ref. 3). In addition to having high continuous wave (CW) power capabilities, plasmas result in moderate harmonic efficiencies. It is apparent from Figure 1 that conventional $\mathrm{CW}$ millimeter wave sources yield less and less power as the frequency of operation is increased. As recently as 1966, the power/frequency frontier was represented by a $-18 \mathrm{~dB}$ /octave asymptote. Thus, if a high-power microwave signal were allowed to interact with a plasma medium in such a manner that the harmonic efficiency would be greater than $-18 \mathrm{~dB} /$ octave, the power/frequency frontier would be advanced.

\section{Harmonic Generation in Gas Discharges}

Unfortunately, the non-linear mechanism responsible for frequency multiplication at high frequencies is not fully understood at this time. Although Margenau and Hartmann (ref. 4) treated this problem theoretically as early as 1948, a number of more recent studies contradict this work. Each of these theoretical treatments considers only a few non-linear mechanisms, such as $\bar{v} \times \bar{B}$ forces (ref. 5), collision frequency modulation (ref. 6), or electric field inhomogeneities (ref. 7), to the exclusion of all others. This is done after a number of simplifying assumptions about the plasma have been made so that the resulting formalism is tractable from a mathematical standpoint. This causes each calculation to have a limited range of validity. Unhappily, the above calculations are hard to verify experimentally because in many instances the model assumed by the theoretician is hard to achieve in practice.

Recent experiments indicate that two non-linear effects discussed by Krentz and Kino (ref. 8) play an important role in harmonic generation in plasmas. The first effect is caused by a static variation in charge density, while the second effect is caused by an inhomogeneity in the electric field. Both effects occur when the plasma number density profile is non-uniform.

Krentz has explained the first effect on a physical basis in the following way. If a spatially uniform, time-varying electric field is applied to a homogeneous plasma, the electrons will have motion that is sinusoidal in time. If, however, the static number density of the plasma has spatial variations, then the field-induced motion of electrons from a region of high density to a region of lower density creates a net timevarying charge density (from the equation of continuity). The product of the rf charge density and the applied electric field amounts to a non-linear term that yields frequency multiplication. 


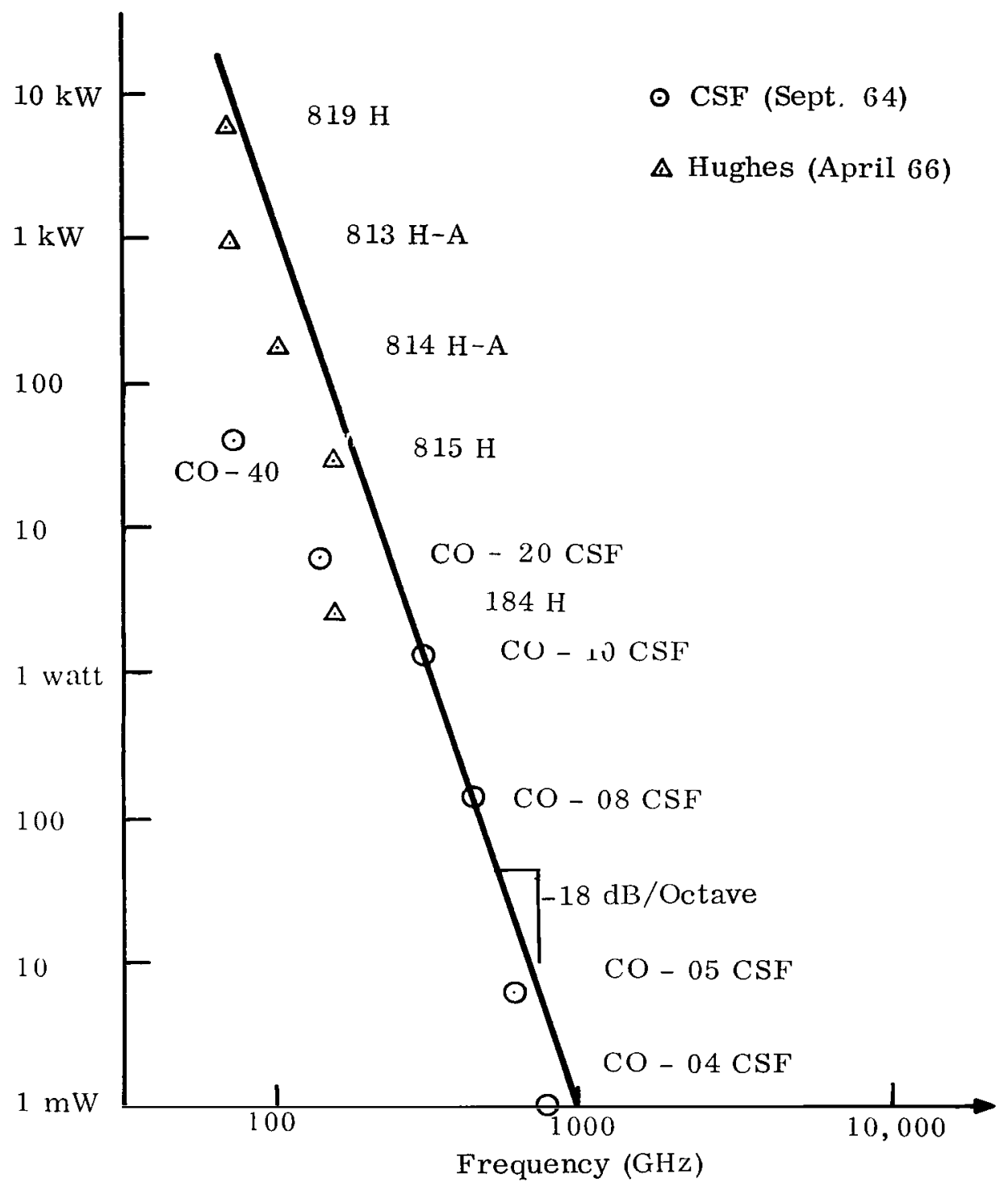

Figure 1. Frontier devices in $\mathrm{cw} \mathrm{mm}$ wave generation (from Kulke and Veronda, ref. 2) 
When the electron number density is uniform but the electric field is not, the second effect is possible. In this case the velocity of the electron is no longer sinusoidal with time for one of two reasons. First, if the field variation is along the direction of the electric field, each electron experiences a field of varying amplitude. Second, if the electric field varies along a direction prependicular to the field, the curl of the electric field will be non-zero, and an rf magnetic field will interact with the moving electrons to give rise to $\overline{\mathrm{V}} \times \overrightarrow{\mathrm{B}}$ forces. Thus, harmonic generation results from any non-uniformity of the electric field.

\section{Experimental Results}

The first investigator to report harmonic generation in a plasma at microwave frequencies was Uenohara (ref. 3) in 1957. His plasma discharge was produced by placing the tips of two cylindrical electrodes close together in a reduced pressure glass cylinder mounted in a rectangular waveguide. An rf-generated plasma was formed when the air in the glass vessel was ionized by the impinging microwave signal. Uenohara was able to obtain a conversion ratio of $-23 \mathrm{~dB}$ between the fundamental $(3 \mathrm{GHz})$ and the second harmonic. He was later able to raise this efficiency to $-16 \mathrm{~dB}$ by increasing the input power and changing the geometry of the electrodes. Other investigators (Baird and Coleman, 1959 (ref. 9), Krentz, 1960 (ref. 10) and Treial, 1961 (ref. 11), have obtained similar results from similar devices (see Table I).

More recently, Kino and Krentz (ref. 8) have obtained large harmonic efficiencies by impinging an input signal at $2.85 \mathrm{GHz}$ on a spherical mercury vapor discharge. They have demonstrated that more than $50 \mathrm{~mW}$ is realizable at the third through seventh harmonics when less than 18 watts of input power was absorbed by the plasma. Unfortunately, the terminal efficiency was considerably less since a large amount of mismatch at the fundamental frequency was necessary to maintain a stable rf discharge. Nevertheless, it was shown that the non-linearities of an rf discharge are sufficient to generate harmonic power with high efficiencies.

Prior to Krentz's work, Swan (ref. 12) had carried out several harmonic experiments with an electrodeless discharge. Although his terminal efficiencies $(-5 \mathrm{~dB}$ at 12 watts input power) were better than those reported by Krentz, he did not generate millimeter waves since his input frequency was only $2.9 \mathrm{GHz}$. Attempts were made by Swan to scale down the dimensions of his generator so that a fundamental frequency of $35 \mathrm{GHz}$ could be used. Unfortunately, these experiments were unsuccessful owing to the fact that air breakdown prevented the coupling of appreciable amounts of power into the discharge region.

Other efforts at scaling down plasma multipliers have been made by Froome (refs. 13-16) and Lauks (refs. 17, 18). Froome was moderately successful in generating frequencies up to $735 \mathrm{GHz}$ when he used a high-pressure arc discharge 
TABLE I

PLASMA MULTIPLIER EXPERIMENTS

(from reference 17 )

\begin{tabular}{|c|c|c|c|c|c|c|c|c|c|}
\hline \multirow[b]{2}{*}{ Year } & \multirow[b]{2}{*}{ Experimenter } & \multicolumn{2}{|c|}{ Fundamental } & \multicolumn{5}{|c|}{ Conversion Efficiency in dB } & \multirow[b]{2}{*}{$n-t h$} \\
\hline & & $\mathrm{GHz}$ & watts & 2 nd & $3 r d$ & 4 th & 5 th & 6 th & \\
\hline 1953 & Hanley \& Ruhlig & 0.2 & 40 & -6.4 & -11 & --- & --- & --- & \\
\hline 1957 & Uenohara & 3 & 12 & -23 & -28 & -43 & --- & --- & \\
\hline 1958 & Whitmer & 10 & $(1)$ & -63 & --- & --- & --- & --- & \\
\hline 1959 & Hill \& Tetenbaum & 3 & $300 \mathrm{p}$ & -15 & -35 & -50 & --- & --- & \\
\hline 1959 & Baird \& Coleman & 10 & $10 \mathrm{kWp}$ & -33 & -43 & -47 & --- & --- & $-53(8 \mathrm{th})$ \\
\hline 1959 & Froome & 2.5 & (5) & --- & --- & -33 & --- & --- & $\mathrm{d}(12 \mathrm{th})$ \\
\hline 1960 & Froome & 35 & 1 & --- & --- & --- & --- & -80 & --- \\
\hline 1960 & Kino \& Krenz & 3 & $(2)$ & -20 & -30 & -40 & -50 & -- & \\
\hline 1960 & Bierrum \& Walsh & 3 & $77 \mathrm{kWp}$ & --- & -35 & --- & --- & --- & \\
\hline 1960 & Uenohara & 3 & 30 & -16 & --- & --- & --- & --- & \\
\hline 1961 & Baird \& Coleman & 10 & 40 & -26 & -30 & -40 & --- & --- & \\
\hline 1961 & Baird \& Coleman & 35 & 25 & -32 & -44 & -60 & --- & --- & \\
\hline 1961 & Treial a. & 2.9 & 17 & -17 & -20 & -35 & --- & --- & \\
\hline 1961 & Treial b. & 2.9 & 15 & -10 & --- & -30 & --- & --- & \\
\hline 1961 & Swan & 2.9 & 12 & -5 & -9 & -12 & --- & --- & $-30(8$ th $)$ \\
\hline 1962 & Froome & 35 & 5 & --- & --- & --- & $-\cdots$ & -52 & $-93(18 \mathrm{th})$ \\
\hline 1963 & Froome & 35 & 10 & --- & --- & --- & --- & --- & d (29th) \\
\hline 1963 & Krenz a. & 3 & 3 & --- & -19 & --- & --- & --- & \\
\hline 1963 & Krenz b. & 3 & 12 & -6.7 & --- & --- & --- & --- & \\
\hline 1963 & Cooke & 9.5 & 9 & -20 & --- & --- & -- & $-\cdots$ & \\
\hline 1963 & Tamaru & 35 & 8 & -35 & --- & --- & --- & --- & \\
\hline 1964 & Knight & 35 & $6 \mathrm{~kW} \mathrm{p}$ & -31 & --- & --- & $-\cdots$ & --- & $-53(7 \mathrm{th})$ \\
\hline 1965 & $\begin{array}{l}\text { Benson, Cooke, } \\
\text { Holmes }\end{array}$ & 9.5 & 10 & -20 & -- & --- & --- & $-\cdots$ & \\
\hline 1965 & Lauks & 3 & 17 & -7.8 & --- & --- & --- & --- & \\
\hline 1965 & Lauks & 34 & 14.7 & -15.2 & --- & --- & --- & $\cdots$ & \\
\hline
\end{tabular}

Note: $p=$ pulsed power (peak value) $\mathrm{d}=$ detected amount of power 
between a tungsten wire anode and a cathode of molten platinum. Although he obtained the highest frequency signal realized up to that time in the submillimeter wave region, recent devices (e.g., CSF Carcinotron, CO-04) have surpassed his generator both in frequency and power.

The plasma multiplier designed by Lauks is shown in Figure 2. The plasma was generated in a glass cylinder by the intense $\mathrm{rf}$ field $(34 \mathrm{GHz})$ that existed between two aluminum electrodes. The separation of the fundamental power and the harmonic power was achieved by an iris which was resonant at the fundamental frequency. By placing this iris the appropriate distance away fron the plasma discharge, the $68-\mathrm{GHz}$ harmonic signal was kept from traveling towards the fundamental power source, and instead was reflected back towards a tapered section leading to a high-frequency waveguide.

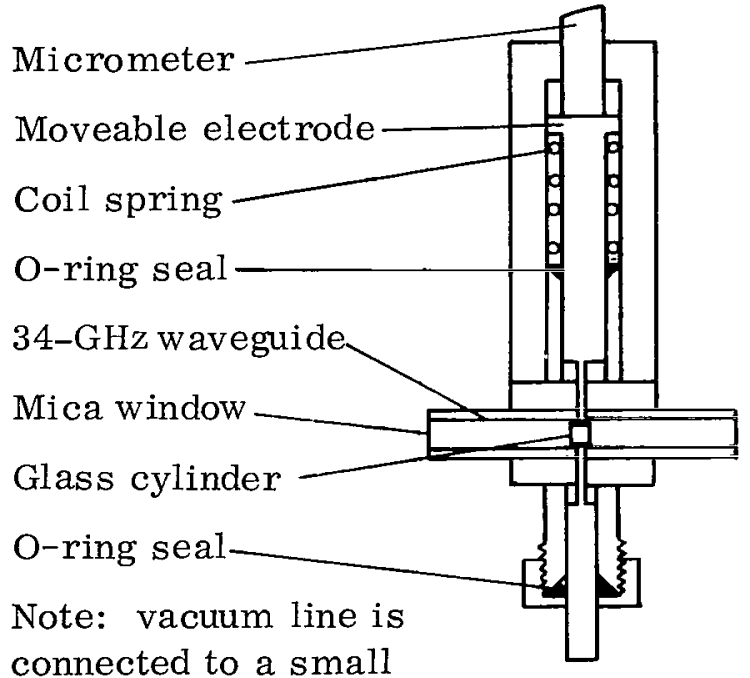

hole in the waveguide scale: full size (the glass cylinder

fits loosely)

Figure 2. Plasma multiplier by Lauks
Lauks observed a power output of 440 $\mathrm{mW}$ at $68 \mathrm{GHz}$, corresponding to a conversion efficiency of $-15.2 \mathrm{~dB}$. This was achieved only after he had tried a large number of electrode shapes, and after he had varied the plasma background pressure $(2-8 \mathrm{~mm} \mathrm{Hg})$ and the size of the glass cylinder that contained the discharge. He found that electrodes approximately 10 mils in diameter inside a 40-mil diameter glass container gave the best results. Unfortunately, operation at this power level endangered the lifetime of the electrodes, and many electrodes were actually destroyed by the heat. Therefore, it might be expected that future scaling of plasma multipliers for operation at higher frequencies would be complicated on account of the heat-transfer problems involved. In addition, the creation of dense plasmas will be necessary if the generation of higher frequencies is to result from future exploration

in this area. More about this contention will be covered in section IV.

At the present time, the investigation of harmonic generation in plasmas is being conducted from an academic point of view. Asmussen and Beyer (ref. 19) at the University of Wisconsin have devised a plasma tripler that yields $600 \mathrm{~mW}$ at $9 \mathrm{GHz}$. McIntosh (ref. 20) has studied harmonic generation from a plasma column in an S-band waveguide at $\mathrm{X}$-band $(7.5 \mathrm{GHz})$. The operation of these devices is nowhere near the power/frequency frontier of millimeter waves. Nevertheless, studies such as these are continuing so that a better understanding of the non-linear mechanisms in a plasma may be obtained. 


\section{ELECTRON BEAM PLASMA DEVICES}

In recent years, many people have speculated on the future application of the beam plasma interaction in microwave and millimeter wave tubes. The amplifying and oscillatory effects that are obtained from this interaction have been investigated in an attempt to establish whether future effort in this area will yield useful results. Already prototype beam plasma tubes have been constructed for operation in the microwave frequency $r$ ange. However, the development of millimeter wave tubes has lagged because of a number of unsolved engineering problems (see section IV).

As early as 1925, Langmuir (ref. 21) proposed that the interaction of an electron beam and a plasma should result in the generation of high-frequency oscillation. It was not until 1948, however, that engineers utilized this concept to explain the spurious oscillations that were being observed in some microwave tubes (ref. 22). At that time, Haeff (ref. 23) demonstrated that, when two coupled electron streams move at different velocities, an amplification results. His analysis was important because the beam plasma interaction is a particular case of the double-stream amplifier mechanism.

In 1949, Bohm and Gross (ref. 24) calculated the electrostatic instabilities in a beam plasma interaction. They used Maxwell's equations and the Lorentz force equation to determine the interaction between plasma electrons with thermal motion and beam electrons whose motion is in one direction only. By clarifying the physical processes of the interaction, they were able to describe the resulting energy transfer between the beam and the plasma. They also considered the effect that finite bounaries have on these oscillations. More recently, Allen and Kino (ref. 25) have analyzed the beam plasma interaction in a cylindrical system in the presence of a finite, axial, magnetic field. By a linear analysis, they were able to obtain numerical solutions of the problem. As this approach is rather involved, a number of people have analyzed this system by making a few simplifying assumptions (e.g., weak coupling, quasistatic). The se simplified approaches allow a direct comparison between the beam plasma interaction and the beam circuit interaction of conventional microwave tubes.

Briggs (ref. 26) has prepared a summary of the theoretical aspects of electron stream interaction with plasmas in a research monograph. This work describes the effects that boundaries, temperature, and magnetic field have on the interaction. Furthermore, Briggs has rigorously determined the nature of the instabilities of this interaction and has categorized them as either convective (growing in space) or absolute (growing in time). He has identified beam plasma interactions that are analogous to both the traveling-wave amplifier and the backward wave oscillator modes of a conventional beam circuit device. 
Additional theoretical studies are being carried on at this time. For instance, Poeschel (ref. 27) has considered the non-linear behavior of a velocity-modulated electron beam interacting with a plasma. Rowe (ref. 28) is also investigating the nonlinear aspects of the beam plasma interaction. Otteni (ref. 29) has considered the effect that collisions and temperatures of both the beam and the plasma have on the interaction. These recent studies are aimed at refining existing theories to improve the correlation with experiments.

\section{Beam Plasma Interaction}

All of the theoretical treatments of the beam plasma problem have one feature in common: the geometrical configuration in which the interaction takes place is assumed to be simple. This is necessary because more complicated structures are simply not tractable from a mathe matical standpoint. Apel (ref. 30) has obtained an approximate dispersion relationship for the system shown in Figure 3 . In this system, the electron stream and plasma fill the entire waveguide. In equilibrium, the plasma electrons have randomly directed velocities, whereas the beam electrons have a velocity component along the axis only, and there is a dc magnetic field in the axial direction.

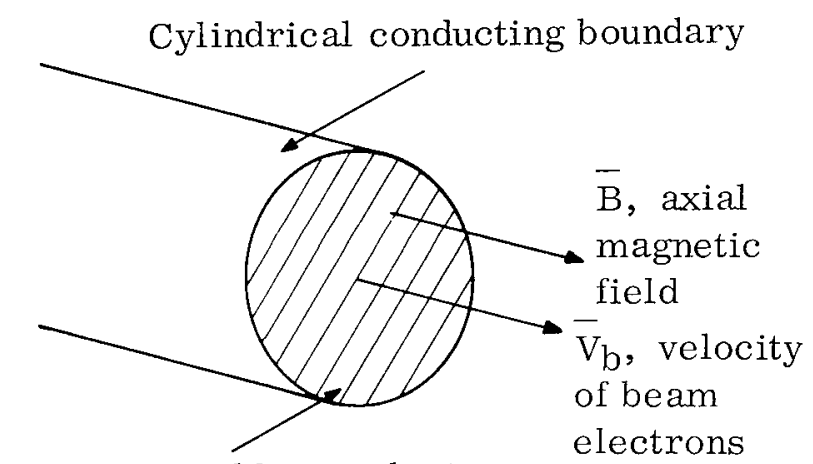

Plasma and beam electrons

Figure 3. Beam plasma interaction treated by Apel
A dispersion diagram that shows the growth or attenuation constant of the most important waves in the above system appears in Figure 4. Although there are many other wave possibilities, it has been demonstrated that the interactions between the slow space charge wave of the beam and the longitudinal wave or the cyclotron wave in the plasma yield the greatest amplification in the beam. Figure 4 shows that the gain constant (imaginary part of the wave number) has two negative maxima $\left(\omega / \omega_{p}=\right.$ 0.28 and $\left.\omega / \omega_{p}=0.96\right)$. The wave growth at the lower frequency has been attributed to the interaction between the "transverse" plasma cyclotron wave and the "longitudinal" beam wave. At the higher frequency, growth results from an interaction between the longitudinal beam and plasma waves.

This interaction is merely the two-stream instability in a system of finite geometry, temperature, and magnetic field. At frequencies higher than the plasma frequency, oscillations in the beam plasma system may be obtained. This effect cannot be seen 


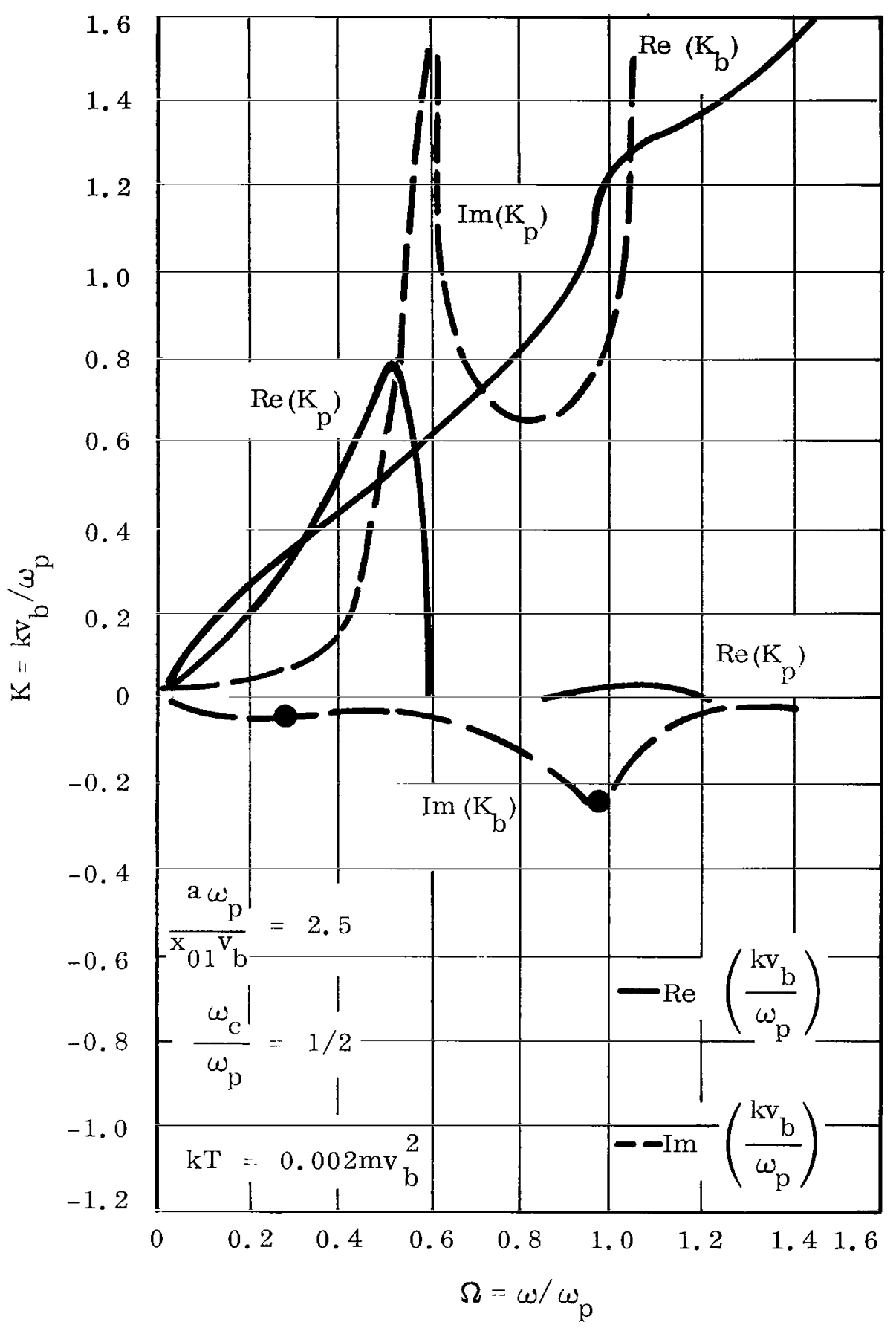

Figure 4. Complex-K roots to the dispersion equation with coupling taken into account. (The solid curves represent the real part of the wave number whereas the dashed lines represent the imaginary part. Subscript $p$ stands for plasma wave $\left(\mathrm{e} . \mathrm{g} ., \mathrm{K}_{\mathrm{p}}\right.$ is the plasma wave number) and subscript $b$ stands for electron beam wave. Note that the phase and group velocities of waves with roots $K_{p}$ and $K_{b}$ are nearly the same in the vicinity of the dots. 
in Figure 4, because absolute instabilities (oscillation growing in time) are properly described by determining the complex frequency as a function of real wave number (ref. 30, p. 28). Both the space charge wave and the slow cyclotron waves of the beam can interact with the plasma waves to cause $\mathrm{rf}$ oscillations analogous to those obtained in a backward-wave oscillator.

A difficulty in solving the problem by computer, as Apel has done, is that most of the physical aspects of the interaction are obscure. At this time, a precise picture of the energy exchange between the beam and the plasma does not exist. This interaction is unlike the beam circuit interaction because it is not so easy to describe the electron bunching in the beam by a simple physical argument.

Kino (ref. 31) gives a heuristic argument in which he shows that growing waves in the electron beam are possible if one notes that the macroscopic permittivity of the plasma medium is negative at frequencies that are less than the electron plasma frequency. He contends that in this case the Coulomb forces act in a direction opposite to their normal sense so that particles of the same species attract (within limits) rather than repel each other. This causes an instability in the beam that is manifested in the amplification of signals on the beam. Although this description is appealing because of its simplicity, it attempts to account by a macroscopic argument for the microscopic behavior of the electrons, and one may question such an extension.

\section{Experimental Results}

As in the case of the plasma harmonic multiplier, the experimental work in beam plasma interactions has been done at microwave frequencies. It is hoped that the development of devices in this frequency range will lead to the eventual development of beam plasma millimeter wave tubes.

Microwave tube engineers are interested in the beam plasma interaction because it seems to offer advantages over conventional beam circuit coupling. For instance, when the amplification mechanism at plasma resonance (longitudinal beam wave-longitudinal plasma wave) is utilized, certain distinct advantages result. First large gains can be achieved over very short interaction distances. Second, this gain is electronically tunable over wide frequency ranges because the electron number density of the plasma can be controlled externally. Third, as the plasma replaces the slow wave circuit of a conventional tube, a delicate metallic interaction structure is not necessary. Therefore, the design of beam plasma tubes would be facilitated in that the interaction circuit would not have the stringent tolerance requirements that slowwave circuits in millimeter wave tubes would have. In addition, the heat transfer problems resulting from these tiny circuits would be absent. 
It has been shown that coupling of rf energy directly from the plasma wave is possible (ref. 32). However, this method of coupling has not proven to be as efficient as had been expected. Therefore, present beam plasma tubes have utilized standard couplers such as helices and cavities. If efficient coupling could be achieved in future beam plasma tubes, a very great advantage would be achieved, because the couplers do not have to be in the immediate vicinity of the electron beam.

Longitudinal Interaction -- Most experiments that have been performed on beam plasma amplifiers utilized the interaction of longitudinal beam and plasma waves at plasma resonance. Boyd, Field and Gould (ref. 33) used this interaction in their original experiments. They obtained significant amplification at 2.5 to $3.5 \mathrm{GHz}$ from a beam plasma amplifier (BPA) in a mercury discharge plasma. Since then, many people have achieved amplification using this mode (see Table II) at other frequencies and using other plasma generation schemes. In most of the recent experiments, a magnetic field was used to confine the electron stream.

Chorney and his co-workers (ref. 34) have been active in the development of highpower beam plasma amplifiers that operate near the plasma frequency. They have also considered the BPA for submillimeter wave amplification. Although they have not built a device that operates in this frequency range, the group has worked on a number of problems that would have to be solved before effective millimeter wave amplifiers

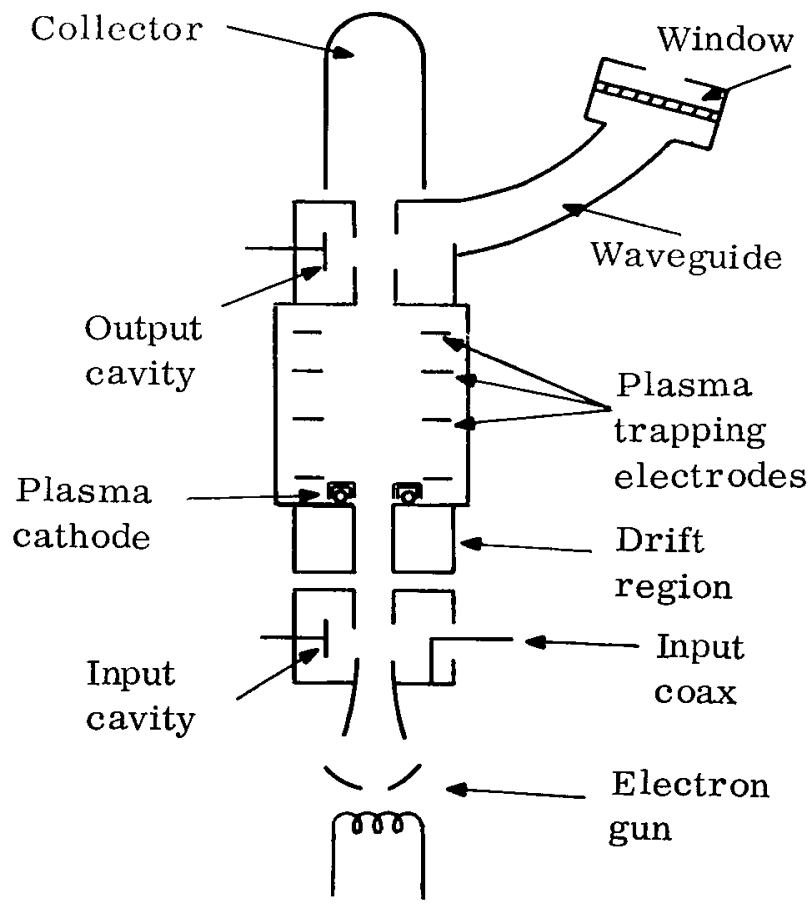

Figure 5. Experimental beam plasma amplifier at $\mathrm{S}$-band could be made. Specifically, they are attempting to couple the input and output signals to the plasma waves rather than to the electron stream. They have also studied methods of generating highly ionized plasmas (ref. 35). These new plasmas have number densities that are large enough and stable enough for interactions at millimeter wave frequencies. An operational microwave BPA designed by Chorney is shown in Figure 5. This amplifier appears to be very similar to standard linear beam tubes in that input and output couplers are placed on either side of the interaction region. The couplers are typical 3-GHz reentrant cavities, and they couple to the space charge waves of the beam. The interaction region is merely a drift tube filled with a xenon plasma at approximately $3 \times 10^{-5}$ torr. The plasma is beam-generated as the ionization process results from the collision of beam electrons with unionized gas atoms. Chorney made some diagnostic experiments on this plasma and 
TABLE II

BEAM PLASMA EXPERIMENTS

\begin{tabular}{|c|c|c|c|c|}
\hline Year & Experimenter & Frequency & Gain & Plasma \\
\hline 1961 & Boyd, Field \& Gould & $2.5-3.5 \mathrm{GHz}$ & - & $\begin{array}{l}\text { Hg discharge } \\
\text { Hydrogen }\end{array}$ \\
\hline 1962 & Curtis \& Ferrari & $7-11 \mathrm{GHz}$ & $60 \mathrm{~dB}$ & $\begin{array}{l}\text { (beam - } \\
\text { generated) }\end{array}$ \\
\hline 1962 & $\begin{array}{l}\text { Vlaardingerbroek \& } \\
\text { Weimer }\end{array}$ & $4 \mathrm{GHz}$ & $30 \mathrm{~dB}$ & Hg discharge \\
\hline 1962 & $\begin{array}{l}\text { Allen, Kino, Spalter, } \\
\text { and Stover }\end{array}$ & $2-4 \mathrm{GHz}$ & $20-30 \mathrm{~dB}$ & $\begin{array}{l}\text { Cesium } \\
\quad \text { (contact) }\end{array}$ \\
\hline 1963 & Kino \& Gerchberg & $450 \mathrm{MHz}$ & $25 \mathrm{~dB}$ & $\mathrm{Hg}$ discharge \\
\hline 1964 & $\begin{array}{l}\text { Eastman, Kerr, \& } \\
\text { Narayan }\end{array}$ & $3 \mathrm{GHz}$ & $20 \mathrm{~dB}$ & $\begin{array}{l}\text { Hydrogen } \\
\text { discharge }\end{array}$ \\
\hline 1964 & $\begin{array}{l}\text { Allen, Biechler, \& } \\
\text { Chorney }\end{array}$ & $3.115 \mathrm{GHz}$ & $25 \mathrm{~dB}$ & $\begin{array}{l}\text { Xenon } \\
\text { (beam- } \\
\text { generated) }\end{array}$ \\
\hline 1964 & Chorney \& Fitzgerald & $11 \mathrm{GHz}$ & $25 \mathrm{~dB}$ & PIG* Xenon \\
\hline 1966 & Apel & $945 \mathrm{MHz}$ & - & PIG Hydrogen \\
\hline *Penn & $\begin{array}{l}\text { Chorney } \\
\text { onization gauge }\end{array}$ & $3 \mathrm{GHz}$ & $55 \mathrm{~dB}$ & $\begin{array}{l}\text { Beam genera- } \\
\text { ted in xenon } \\
\text { gas }\end{array}$ \\
\hline
\end{tabular}

found that the number density profile in the axial and radial directions agrees with the theory. Furthermore, knowledge of the local plasma frequency as a function of position is useful in interpreting his experimental results.

Chorney obtained 55-dB gain for low output powers (less than $5 \mathrm{~kW}$ ) from the above BPA. The device saturated at higher power levels, and a maximum output power of $73.5 \mathrm{dBm}$ was achieved. In obtaining these high power outputs it was necessary to adjust the plasma conditions, because the $\mathrm{rf}$ fields on the beam play an important part in the plasma generation. Therefore, such parameters as gas pressure and electrode trapping voltages were varied in an attempt to optimize efficiencies. As a result of these efforts, Chorney has shown that BPA's can handle high powers at high efficiencies. Challenging problems have to be solved, however, before the BPA can be operated at millimeter wave frequencies. The most important of these is the coupling problem. Until someone learns how to couple millimeter wave power into 
and out of this device efficiently, it will continue to suffer from the disadvantages of fabrication and beam control that are inherent in conventional linear beam tubes.

Tranverse Interactions -- A few investigators have studied the possibility of using transverse modes to obtain amplification from the beam plasma interaction. Kino and Gerchberg (ref. 31) have obtained 25-dB gain from a device similar to Chorney's except thit the input and output signals were coupled to the electron beam in a direction perpendicular to the beam's axis. By doing this, azimuthally varying modes of interaction were utilized in achieving gain at $450 \mathrm{MHz}$. More recently, Crawford (ref. 36) has continued work in the area of transverse wave interactions. He has attempted to couple a bipolar field to the electron stream at the Tonks resonance. However, this technique has not been successful as yet.

Ferrari (ref. 37) has also tried a similar modulation scheme. However, an interaction at the cyclotron resonance was used in his device. Such an interaction holds only limited promise for use at millimeter wave frequencies because of the high magnetic field required. To eliminate these stringent magnetic field requirements, it has been suggested that interactions at harmonics of the cyclotron resonance might be used.

It is not known whether anyone has amplified an $\mathrm{rf}$ signal by using this technique. Nevertheless, work is being carried out at the Sperry Rand Research Center (ref. 38) and at the University of Michigan (ref. 39) to investigate experimentally the possibilities of this and other interactions. Most of the experimental work to date has been concerned with the amplification of signals in a beam plasma system. The apparent philosophy of this approach is that once competitive amplifiers have been developed, efficient oscillators could be designed using appropriate feedback schemes. Some investigators have observed spontaneous (electron) oscillations that were related to the electron cyclotron frequency (i.e., at frequencies that depended on the magnetic field intensity). Agdur (ref. 40) reported similar results when he injected an electron stream into an argon plasma. He observed spontaneous oscillations that occurred near the electron cyclotron frequency suggesting that the backward-wave interaction was responsible. However, the nature of these oscillations is not completely understood. In some experiments other effects, not included in the theory, can introduce feedback into the system. 


\section{CONCLUSIONS}

This Technical Note has described two possible ways of generating millimeter waves. Although a plasma is used in both cases, the operation of the proposed devices is distinctly different. The plasma multiplier utilizes the non-linear properties of a plasma to achieve harmonic multiplication, whereas the beam plasma amplifier depends on the inductive nature of a plasma. However, efficient operation at millimeter wave frequencies has not been achieved for these devices on account of difficulties common to both.

Microwave plasma multipliers and BPA's have operated most efficiently at frequencies that are near the electron plasma frequency. Therefore, it can be expected that at higher frequencies denser plasmas that should be relatively free of random fluctuations and spontaneous oscillations will be required. Moreover, these quiescent plasmas should be stable over reasonably long periods of time.

Until the coupling problem of current BPA's has been solved, circuit scaling is necessary in each case. Both techniques of generating millimeter waves are limited by mechanical tolerance and heat-dissipation problems. Even if the materials and machining problems could be solved, the solution of other engineering problems would be necessary. For example, thermal expansion could cause serious detuning of both kinds of generators. In the following paragraphs, the problem areas of plasma millimeter wave generation are described in more detail.

\section{Plasma Harmonic Generator}

Very little experimental work at millimeter wavelengths has been done using plasma harmonic multipliers. Therefore, it is necessary to appraise the potential of these devices at $\mathrm{mm}$ wavelengths on the basis of their performance at microwave frequencies. C. B. Swan of Bell Telephone Laboratories, Inc., has noted that there are three major aspects to the problem of operating these devices at high frequencies. The problems to be solved are:

1. The generation of plasma with the desired properties

2. The scaling of circuits

3. The elimination of the inherent difficulties of maintaining a gas discharge. 
Swan has shown that if harmonic generation in a plasma is caused by field gradients (see section II), then the efficiency of the conversion process is unaffected by dimensional scaling so long as similar gas discharges are maintained. This means that the electron density must increase as the square of the frequency, while the gas pressure increases directly with frequency. Thus, it will be necessary to obtain a highly ionized plasma if efficient $\mathrm{mm}$ wavelength generation is to result. At this time it is not known if $\mathrm{rf}$-generated plasmas that have the required number densities can be achieved.

The circuit scaling problems associated with high-frequency multipliers are more severe. If the dimensions of the multiplier are linearly scaled as $1 / f$, the heat dissipation per unit surface area would increase as $\mathrm{f}^{2}$. The destruction of structures that are in intimate contact with the plasma can therefore be expected. Indeed, Lauks has found in his generator that the electrodes and the discharge containment vessel are excessively heated in operation. He has also observed that sputtering is an ever-increasing problem at $\mathrm{mm}$ wavelengths because of the invariance of the total sputtering rate when the generator is scaled down in size.

In nearly all efficient microwave plasma harmonic multipliers, the fundamental power sustains the discharge. Unfortunately, the fundamental signal is not matched to the discharge region prior to the initiation of the $\mathrm{rf}$ discharge. Thus, it is usually necessary to start the discharge with an auxiliary source. Lauks has been successful in starting his discharge by changing the distance between two electrodes. This method is cumbersome, and an improved technique would alleviate this serious disadvantage. For this reason, rf-generated plasmas present difficulties if pulse operation is envisaged.

Another undesirable feature of recent prototype multipliers is the necessity of controlling the gas pressure by continuous pumping, as this is the only feasible method of avoiding unwanted pressure variations. If one adds to the list of shortcomings that such generators tend to be quite noisy at moderate power levels, then it is difficult to be optimistic about the use of these devices at $\mathrm{mm}$ wavelengths.

Additional disadvantages of the plasma harmonic generator might be determined if further development in this area takes place. For example, measurements of the FM noise contributed to a system utilizing a plasma multiplier have not been made. The lack of such important data suggests that the development of this device is in its early stages. If one considers that plasma multipliers have been studied for more than 10 years, he is impressed with the lack of progress in this area. Solid-state plasmas are expected to be better suited for the harmonic generation of submillimeter wavelengths. 


\section{Beam Plasma Interaction Devices}

Investigations of beam plasma interaction have been conducted for nearly the same length of time as those of the plasma harmonic multiplier. During this period, many of the original expectations have been realized, and devices using the beam plasma interaction could find application. It has not yet been established whether such interactions will be helpful in the amplification and generation of $\mathrm{mm}$ waves. Four major problem areas exist at present where answers must be found before progress can be made.

As for the plasma multiplier, a significant roadblock to the extension of beam plasma interactions into the millimeter wave range lies in the generation of appropriate plasmas. At these high frequencies, it will be necessary to generate a dense plasma. It is also desirable that the percentage of ionization of this plasma be high, since the $\mathrm{rf}$ signal on the beam tends to ionize neutral atoms in the plasma. This last effect is important if pulsed operation is expected as it may limit the peak $\mathrm{rf}$ power on the beam.

The PIG (Penning ionization gauge) discharge has been a widely used method for obtaining a plasma for microwave BPA's. This discharge is well-suited for $\mathrm{mm}$ wave applications, as it can operate at low pressures and a high percentage of ionization. Chorney has been successful in obtaining PIG discharges with a plasma frequency of $160 \mathrm{GHz}$. Other methods of plasma generation, such as the contact ionization of cesium and beam generation, have been used. Further studies of plasma generation are going on at Microwave Associates, Inc. (ref. 34), the University of Utah (ref. 41), and at the U.S. Army Electronics Command, Fort Monmouth, N.J. (ref. 42). The problem of random fluctuations and oscillations is of critical importance in the beam plasma device. Unless the beam plasma amplifier operates at high enough power levels, the noise contributed to the signal by the plasma will result in a poor overall noise figure. Furthermore, a host of natural oscillations can be set up by the interaction of the beam with the plasma. These instabilities generally occur at low frequencies and are caused by ion motion in the plasma; they must be suppressed when their presence affects the performance at higher frequencies.

Perhaps the most important problem that must be solved before beam plasma interaction can be used in the millimeter wavelength range is that of coupling directly to the plasma waves. Such coupling of energy will obviously be necessary if present $\mathrm{BPA}^{\prime}$ 's are to be operative at these wavelengths.

Early experiments performed by Chorney (ref. 32) show that coupling directly to the plasma can be relatively efficient. In coupling to the plasma he has obtained a terminal gain of $-8 \mathrm{~dB}$ from a BPA operating at $2.8 \mathrm{GHz}$. He has noted that the plasma density that gives maximum electronic gain is not the same as that which gives maximum terminal gain when the coupling of plasma waves is used, because the plasma 
density in the vicinity of the electron beam is responsible for large gains, whereas the plasma density outside the electron beam is important in efficient coupling.

Chorney's more recent experiments have been more successful (ref. 43). He utilized two different plasma regions in one amplifier and thereby was able to adjust one plasma region for large electronic gain and the other for efficient coupling. With this arrangement, a net terminal gain of $+18 \mathrm{~dB}$ was achieved at S-band. This technique has not been tried at millimeter wave frequencies. Rather than using a conventional reentrant cavity or a waveguide coupling scheme, Rowe (ref. 44) investigated the possibility of coupling to a beam plasma system with an elliptical cavity coupler so designed that the beam plasma interaction takes place at one focus of an elliptical cavity and an output probe is located at the other focus. Such an arrangement shows promise as a future coupling scheme in beam plasma devices.

The last problem area is that of electron beam formation. Although certain beam requirements are less severe (e.g., beam diameters can be larger), other aspects of beam generation are complicated owing to the presence of a plasma in the system. The life of the gun cathode will, in general, be reduced if heavy ions are allowed to bombard the cathode. Drainage holes and trapping electrodes are therefore necessary to protect the electron gun.

To date, harmonic generators and amplifiers using a plasma have not been so successful as other methods of generating millimeter waves. If these devices are to become competitive with other generation techniques, solutions of the problems described in the preceding section are necessary. Many of these problems will probably be eliminated as the overall tube and plasma technologies advance. Therefore, the competitive position that generators using plasmas may have in the future is worth considering.

With the assumption that the plasma generation problems can be eliminated, there still remains the problem of maintaining a gaseous plasma. This may require voltage supplies, or other supporting equipment, that are heavy and costly. Furthermore, methods of strictly controlling ambient conditions will have to be provided in applications of a critical nature, because the pressure of a gaseous discharge is dependent on temperature. Finally, the noise figure of such devices can be expected to be poor at low power, and at high-power levels distortion will occur if the amplifier or multiplier is used for short pulse applications.

Perhaps one can justify further effort in beam plasma studies when power handling capability is considered. Large rf power output has already been demonstrated from beam plasma amplifiers. It should be noted, however, that plasma harmonic generators are losing much of the power handling edge that they once had over solidstate varactors as a result of the large development programs in the solid-state area. (Present varactor multipliers can handle watts at $\mathrm{X}$-band.) Continuing studies of 
plașma interactions for application in millimeter wave generation might be warranted at this time. * Past studies have already contributed to the understanding of conventional tube operation and could have an impact on future solid-state plasma research.

* Since considerable promise has been shown. But it must be kept in mind that, although feasibility has been shown in many cases, there remain a considerable number of practical problems that have yet to be overcome. 


\section{REFERENCES}

1. Forster, D. C.: High Power Sources at Millimeter Wavelengths. Proc. I.E.E.E., Vol. 54, No. 4, pp. 532-539, April 1966.

2. Kulke, B., and Veronda, C. M.: Millimeter-Wave Generation with Electron Beam Devices. Microwave J., Vol. 10, No. 10, pp. 45-53, September 1967.

3. Uenohara, M. : Uenohara, M., Masutani, T., and Inada, K.: A New HighPower Frequency Multiplier. Proc. I. R. E., Vol. 45, No. 10, pp. 14191420, October 1957.

4. Margenau, H., and Hartman, L. M.: Theory of High-Frequency Gas Discharges--II - Harmonic Components of the Distribution Function. Phys. Rev., Vol. 73, No. 4, pp. 309-328, February 1948.

5. Tang, T. W.: Harmonic Generation by an Electromagnetic Wave in an Inhomogeneous Isotropic Plasma. I. E.E.E. Trans. on Antennas and Propagation. Vol. AP-14, No. 1, pp. 54-62, January 1966.

6. Murphy, B.: Harmonic Generation in a Microwave Discharge. Phys. Fluids, Vol. 8, No. 8, pp. 1534-1540, August 1965.

7. Chen, Kun-Mu: Interaction of a High Intensity EM Field with a Low-Density Plasma, I. R.E. Trans. on Antennas and Propagation, Vol. AP-10, No. 1, p. 31, January 1962 .

8. Krentz, J. H., and Kino, G. S.: Harmonic Generation and Parametric Oscillations in a Plasma. J. Appl. Phys., Vol. 36, No. 8, pp. 2387-2395, August 1965.

9. Baird, J. R., and Coleman, P. D.: High-Power Gas Discharge Frequency Multiplier. Proc. Symp. on Millimeter Waves, New York, N. Y., April, 1959, Polytechnic Press, Brooklyn, N. Y., 1960.

10. Krentz, J. H.: Harmonic Generation in Plasmas. Ph.D. Thesis, Stanford University, 1963; Microwave Laboratory Report No. 1055, Stanford University, 1963.

11. Treial, H.: Microwave Harmonic Generation by Electron Density Modulation in a Plasma. MA Sc. Thesis, Dept. of Elect. Engng., Univ. of Toronto, Toronto, Canada, January, 1962. 
12. Swan, C.B.: Generation of Microwave Harmonics in an Electrode-less Discharge at Low Pressure. Proc. I. R. E., Vol. 49, p. 1941, 1961.

13. Froome, K. D.: A New Microwave Harmonic Generator. Nature, Vol. 184, No. 4689 , p. 808 , September 1959.

14. Froome, K. D.: Millimeter Waves from Mercury Arc Harmonic Generator. Nature, Vol. 186, No. 4729, p. 959, June 1960.

15. Froome, K. D.: Sub-millimeter Waves by Harmonic Generation from Cold Cathode Arcs. Nature, Vol. 188, No. 4744, pp. 43-44, October 1960.

16. Froome, K. D.: Microwave Harmonic Generator Capable of Frequencies in Excess of $600 \mathrm{Gc} / \mathrm{s}$. Nature, Vol. 193, No. 4821, pp. 1169-1170, March 1962 .

17. Lauks, V.: Millimeter Wave Harmonic Generation in a Plasma. Research Report No. 37, Dept. of Elect. Engng., Univ. of Toronto, Toronto, Canada, November 1965.

18. Yen, J. L., and Lauks, V.: Plasma Harmonic Generation at Millimeter Wavelengths. Electronics Letters, Vol. 2, pp. 20-21, January 1965.

19. Asmussen, J.: Experimental Investigation of Microwave Harmonic Generation in a Plasma, Ph. D. Thesis, Dept. of Elect. Engng., University of Wisconsin, Madison, Wisconsin, June 1967.

20. McIntosh, R. E.: Secular Effects as Related to the Nonlinear Behavior of Plasmas. Ph. D. Thesis, Dept. of Elect. Engng. Univ. of Iowa, Iowa City, Ia., August 1967.

21. Langmuir, I.: Scattering of Electrons in Ionized Gases. Phys. Rev., Vol. 26, No. 5, pp. 585-613, November 1925.

22. Pierce, J. R.: Possible Fluctuations in Electron Streams due to Ions. J. Appl. Phys. Vol. 19, No. 3, pp. 231-236, March 1948.

23. Haeff, A. V.: Space-Charge Wave Amplification Effects. Phys. Rev., Vol. 74, No. 10, pp. 1532-1533, November 1948.

24. Bohm, D., and Gross, E. P.: Theory of Plasma Oscillations, Part A--Origin of Medium-like Behavior. Phys. Rev., Vol. 75, No. 12, pp. 1851-1863, June 1949; Part B--Excitation and Damping of Oscillations. Phys. Rev., Vol. 75, 
No. 12, pp. 1864-1876, June 1949; Part C--Effect of Plasma Boundaries on Oscillations. Phys. Rev., Vol. 79, No. 6, pp. 992-1001, September 1950.

25. Allen, M. A., and Kino, G. S.: Interaction of an Electron Beam with a Fully Ionized Plasma. Phys. Rev. Letters, Vol. 6, No. 4, pp. 163-165, February 1961.

26. Briggs, R. J.: Electron Stream Interactions with Plasmas. M. I. T. Press, Cambridge, Mass., 1964.

27. Poeschel, R. L.: A Nonlinear Study of Beam Plasma Amplification. Tech. Report No. 33 (Nonr 220(50), ASTIA No. AD 639057), California Inst. of Tech., September 1966.

28. Rowe, J. E., Brackett, C. A., Konrad, G. T., and Lugg, A. M. : Microwave Generation by Means of Interaction with Anisotropic Media. Quarterly Progress Report No. 6 (DA-28-043 AMC 01315(E), ASTIA No. AD 813572), Electron Physics Laboratory, Univ. of Michigan, March 1967.

29. Otteni, G. A.: Wave Interaction in Nonrelativistic Electron Beam-Plasma Systems. Scientific Report No. 17 (AF19(628)1699), Case Inst. of Tech., July 1967.

30. Apel, J. R.: Studies of Beam-Plasma Interactions in a Magnetic Field. Tech. Memo. (NOw 62-0604-c, ASTIA No. AD 644695), Appl. Phys. Lab., Johns Hopkins Univ., June 1966.

31. Kino, G. S., and Gerchberg, R.: Transverse Field Interactions of a Beam and Plasma. Proc. VIth Int. Conf. on Ionization Phenomena in Gases, Paris, France, July 1963; Serma Publishing Co., Paris, France, Vol. 3, pp. 219$222,1963$.

32. Allen, M. A.; Biechler, C. S.; and Chorney, P.: Beam-Plasma Amplification for High Power Density Applications. Proc. of the Vth Int. Congress on Microwave Tubes, Paris, France, September 14-18, 1964; Dunod, Paris, pp. 435$438,1964$.

33. Boyd, G. D.; Field, L. M.; and Gould, R. W.: Excitation of Plasma Oscillations and Growing Plasma Waves. Phys. Rev., Vol. 109, No. 4, pp. 13931394, February 1958.

34. Chorney, P.; and Madore, R. J.: Millimeter Wave Component Development (Beam Plasma Amplifier). Final Report (AF30(602)-2948, ASTIA No. AD 609011), Microwave Associates, Inc., April 1965. 
35. Biechler, C. S., Chorney, P., Maddix, H. S., and Madore, R. J.: Generation of Plasmas for Beam-Plasma Amplifiers. Proc. of the Vth Int. Congress on Microwave Tubes, Paris, France, September 14-18, 1964; Dunod, Paris, pp. $441-444,1964$.

36. Crawford, F. W.: Investigation of Fast Wave Beam-Plasma Interactions. Quarterly Rept. No. 3 (DA-28-043 AMC-02041(E), ASTIA No. AD 808347), Inst. for Plasma Research, Stanford Univ., January 1967.

37. Ferrari, R. L.: An Electron Beam-Plasma Amplifier at Microwave Frequencies. J. Electronics and Control, Vol. 17, pp. 49-65, July 1964.

38. Gruber, S.: Investigation of High-Power Beam Plasma Interactions. 4th Quarterly Report (DA-28-043-AMC-01821(E), ASTIA No. AD 812770), Sperry Rand Research Center, March 1967.

39. Getty, W. D.: Frequency Multiplication in High-Energy Electron Beams. Semi-annual Progress Report No. 1 (NASA Grant NGR 23-005-183), Electron Physics Laboratory, Dept. of Elect. Engng., Univ. of Michigan, Ann Arbor, Michigan, May 1967.

40. Agdur, B.: Oscillations in Long Electron Beams, Ericsson Tech., Vol. 16, p. 45, 1960 .

41. Ma, C. H., and Johnson, C. C.: Microwave Device and Physical Electronics Laboratory Consolidated Quarterly Report, Part III--Plasma Studies. (NSF Grant GK 29, ASTIA No. AD 801384), Dept. of Elect. Engng., Univ. of Utah, June 1966.

42. Weiner, M. ; True, R. M. ; and Edwards, E. V.: Investigation of Plasma Generators for Microwave Devices. Tech. Report ECOM-2704, ASTIA No. AD 636956, Electron Tubes Division, Electronic Components Laboratory, June 1966.

43. Chorney, P.: Recent Advances in Beam-Plasma Amplifiers. 1966 I. E. E.E. Convention Record, Part 5, I.E.E.E. Int. Conv., New York, N. Y., March 21-25, pp. 5-16, 1966. 
44. Rowe, J. E., Brackett, C. A., and Konrad, G. T. : Microwave Generation by Means of Interaction with Anisotropic Media. Quarterly Progress Report No. 1 (DA-28-043 AMC-01315 (E), ASTIA No. AD 626196), Electron Phys. Lab., Univ. of Michigan, October 1965.

Electronics Research Center National Aeronautics and Space Administration Cambridge, Massachusetts, October 1967

129-02-03-01-25 
$10 U 00150 \quad 51305 \quad 6805900903$

AIR FORCE WEAPONS LABURATORY/AFWLI

KIRTLAND AIR FORCE BASE, NEW MEXICO 8711

ATT MISS MADELINE F. CANOVA, CHIEF TECHN:

LIBRARY /WLILI

POSTMASTER: Undeliverable (Section 158
Postal Manual) Do Not Return

"The aeronautical and space activities of the United States shall be conducted so as to contribute ... to the expansion of human knowledge of phenomena in the atmospbere and space. The Administration shall provide for the widest practicable and appropriate dissemination of information concerning its activities and the results thereof."

- National aeronautics and Space ACt of 1958

\section{NASA SCIENTIFIC AND TECHNICAL PUBLICATIONS}

TECHNICAL REPORTS: Scientific and technical information considered important, complete, and a lasting contribution to existing knowledge.

TECHNICAL NOTES: Information less broad in scope but nevertheless of importance as a contribution to existing knowledge.

TECHNICAL MEMORANDUMS: Information receiving limited distribution because of preliminary data, security classification, or other reasons.

CONTRACTOR REPORTS: Scientific and technical information generated under a NASA contract or grant and considered an important contribution to existing knowledge.

TECHNICAL TRANSLATIONS: Information published in a foreign language considered to merit NASA distribution in English.

SPECIAL PUBLICATIONS: Information derived from or of value to NASA activities. Publications include conference proceedings, monographs, data compilations, handbooks, sourcebooks, and special bibliographies.

TECHNOLOGY UTILIZATION PUBLICATIONȘ: Information on technology used by NASA that may be of particular interest in commercial and other non-aerospace applications. Publications include Tech Briefs, Technology Utilization Reports and Notes, and Technology Surveys.

Details on the availability of these publications may be obtained from:

SCIENTIFIC AND TECHNICAL INFORMATION DIVISION

NATIONAL AERONAUTICS AND SPACE ADMINISTRATION

Washingion, D.C. 20546 